\title{
Orientación empática de los estudiantes de dos escuelas de kinesiología de Chile
}

\author{
A.M. Rojas-Serey, S. Castañeda-Barthelemiez, R.A. Parraguez-Infiesta
}

Objetivo. Conocer la orientación empática de los alumnos de la carrera de kinesiología de dos escuelas de la región metropolitana. Sujetos y métodos. Este trabajo corresponde a una investigación analítica de corte transversal realizada entre los meses de marzo y noviembre del año 2006. Participaron 274 alumnos de un universo de 351 correspondientes a los niveles I, III y V de la carrera de kinesiología de la Universidad de Chile y la Universidad Mayor. Se aplicó la escala de empatía médica de Jefferson (EEMJ). Para el análisis de los datos, se utilizó la prueba $U$ no paramétrica de Wilcoxon-Mann-Whitney y la prueba no paramétrica de Kruskal-Wallis. Resultados. Se obtuvieron mayores puntuaciones en la EEMJ con significación estadística en el tercer y quinto nivel de la carrera con relación al primero $(p<0,05)$. No se encontraron diferencias significativas en las puntuaciones según sexo. Conclusiones. Existen mayores puntuaciones obtenidas en la EEMJ en los alumnos que están en niveles más avanzados de la carrera de kinesiología, y esta diferencia es significativa en ambas universidades. Las puntuaciones obtenidas en la EEMJ no tienen significación estadística en relación con la variable sexo en ambas universidades.

Palabras clave. Educación médica. Empatía. Escala de empatía médica de Jefferson (EEMJ). Kinesiología. Kinesiterapia. Orientación empática.

Empathetic orientation of the physical therapist's students from two schools of Chile

Aim. To know the empathetic orientation of the physical therapist's students in two schools of the metropolitan region. subjects and methods. This work is an analytical cross-sectional research, carried out between the months on March and November, 2006. 274 out of 351 students participated, who belonged to the levels I, III and V of the Universidad de
Chile and Universidad Mayor Physical Therapy Programs. The Jefferson Scale of Physician Empathy (JSPE) was applied. For data analysis, the non-parametrical Wilcoxon-Mann-Whitney test and the non-parametrical Kruskal-Wallis test were used. Results. Higher scores in the JSPE with statistical significance were obtained in the third and fifth level of the program compared with the first one $(p<0.05)$. No significant differences were found in the scores related to gender. Conclusions. There exists higher scores obtained in the JSPE in students who are in more advanced levels of the Physical Therapy Program, being this difference significant in both universities. The scores obtained in the JSPE do not have statistical significance in relation to the variable gender in both universities.

Key words. Empathetic orientation. Empathy. Jefferson Scale of Physician Empathy. Medical education. Physical Therapy.

\section{Introducción}

La empatía se define como un atributo cognitivo que implica la capacidad de entender las perspectivas y experiencias internas del paciente y la habilidad de comunicar esta comprensión [1-6]. Tiene, además, una gran influencia en el desarrollo óptimo de los individuos, en la creación de refuerzos positivos en las relaciones interpersonales y en la comprensión de las emociones, deseos e intenciones de uno mismo, como así también de otros [7].

La literatura existente informa de que la empatía médica se asocia a una serie de atributos, como el grado de satisfacción y la competencia en la realización de anamnesis, entre otros, lo que lleva en conjunto a tener mejores relaciones terapéuticas y buenos resultados clínicos $[3,5,8]$.
Escuela de Kinesiología Facultad de Medicina. Universidad de Chile. Santiago de Chile, Chile.

Correspondencia Dra. Ana M. Rojas Serey. Escuela de Kinesiología. Facultad de Medicina. Universidad de Chile. Avda. Independencia, 1027 Santiago de Chile, Chile.

E-mail amrojas@med.uchile.cl 
Tabla I. Descripción de los porcentajes de respuesta de la escala de empatía médica de Jefferson en relación con el universo.

\begin{tabular}{lcccc}
\hline & $n$ & Participantes & $\begin{array}{c}\text { Porcentaje de } \\
\text { participantes }\end{array}$ & Sexo (masculino) \\
\hline Nivel I & 140 & 101 & $72,14 \%$ & $30,69 \%$ \\
\hline Nivel III & 119 & 90 & $75,63 \%$ & $31,11 \%$ \\
\hline Nivel V & 9 & 83 & $90,22 \%$ & $34,93 \%$ \\
\hline Total & 351 & 274 & $78,06 \%$ & $32,12 \%$ \\
\hline
\end{tabular}

Sin embargo, existen estudios que describen que gran parte de los reclamos en salud se relacionan con una mala atención profesional. Éstos constituyen más del $20 \%$ de los litigios recibidos en el Servicio Nacional del Consumidor en el período 2004-2005 [9].

Se ha demostrado que existe una disminución en el humanitarismo, entusiasmo e idealismo entre estudiantes de medicina [10], además de trabajos que muestran una disminución de la empatía en el transcurso de las carreras de medicina y odontología $[4,8]$.

Sin embargo, todos estos trabajos han sido realizados en médicos, odontólogos y enfermeras, por lo que existe nula investigación sobre empatía en carreras del área de la terapia física.

El objetivo principal de esta investigación consiste en describir la empatía de los alumnos de kinesiología de dos escuelas de Santiago de Chile, según sexo y niveles de enseñanza, utilizando como instrumento de medición la escala de empatía médica de Jefferson (EEMJ) (Jefferson Scale of Physician Empathy, JSPE), un elemento comúnmente utilizado para este propósito [2,3,6,11-14].

\section{Sujetos y métodos}

Este trabajo corresponde a un estudio de tipo analítico, de corte transversal, realizado entre septiembre y noviembre del año 2006.

\section{Participantes}

Participaron en este trabajo 351 estudiantes de la carrera de kinesiología de los niveles primero, tercero y quinto de la Facultad de Medicina de la Universidad de Chile, y de la Universidad Mayor (Tabla I).

\section{Materiales}

Se utilizó la EEMJ, una herramienta consistente en 20 reactivos tipo Likert en una escala de 7 puntos ( 1 = fuertemente en desacuerdo, 7 = fuertemente de acuerdo), construida sobre la base de una extensa revisión de la literatura, seguida de estudios pilotos y con una amplia evidencia publicada que apoya los indicadores psicométricos de la escala (validez de constructo y consistencia interna), tanto en su versión en inglés como en su versión culturalmente adaptada al español $[2,3,6,11-14]$.

\section{Procedimiento}

Se realizó un estudio piloto en donde se aplicó la EEMJ a 15 alumnos de la carrera de kinesiología de los niveles no participantes en el estudio, correspondientes a segundo y cuarto año $(n=30)$ de la Facultad de Medicina de la Universidad de Chile, elegidos al azar. Se entregó a los alumnos la versión en español del instrumento para que lo respondieran en un encuentro cara a cara con uno de los investigadores. Se registraron consul- 


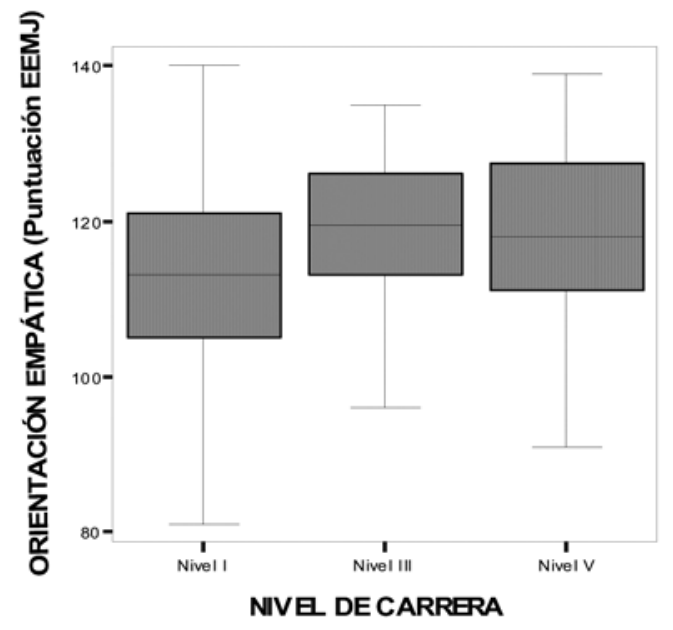

Figura 1. Puntuación obtenida en la escala de empatía médica de Jefferson respecto al nivel de carrera. Se observa que existen mayores puntuaciones obtenidas en los cursos superiores.

Tabla Il. Mediana y rango (mínimo y máximo) obtenidos por nivel.

\begin{tabular}{lcc}
\hline & Mediana & Rango \\
\hline Nivel I & 113 & $81-140$ \\
\hline Nivel III & 119,5 & $96-135$ \\
\hline Nivel V & 118 & $91-139$ \\
\hline
\end{tabular}

tas de ayuda e interpretación de la EEMJ para verificar la comprensión del instrumento.

Posteriormente, se entregó a los alumnos participantes la versión en español de la EEMJ en su versión para estudiantes (versión S). Se realizó una medición única en la sala de clases a todos los alumnos presentes de cada nivel. La información entregada a los alumnos la proporcionó siempre el mismo investigador, utilizando siempre el mismo discurso. Se aseguró a los estudiantes que iban a responder la prueba su confidencialidad. Para el caso particular de los alumnos de quinto nivel de la Universidad de Chile que no comparten un espacio físico común durante el año, se entregó la escala en una visita a cada uno de los centros donde ellos se desempeñan en su práctica profesional, con las mismas indicaciones que para los cursos anteriores.

\section{Análisis estadístico}

Este análisis no tuvo como propósito la comparación de puntuaciones entre las dos universidades, sino entre los distintos niveles de formación. En el análisis estadístico se utilizó la prueba no paramétrica de Kruskal-Wallis para el estudio de las puntuaciones obtenidas en la EEMJ entre los tres niveles de carrera.

Para la comparación de las puntuaciones entre los sexos dentro de cada nivel, se utilizó la prueba $U$ no paramétrica de Wilcoxon-Mann-Whitney.

Se trabajó con un nivel de significación $\alpha=0,05$. Se usó el programa computacional estadístico SPSS v. 15.0.

\section{Resultados}

Las puntuaciones obtenidas en los diferentes niveles en ambas universidades variaron entre un mínimo de 81 y un máximo de 140 puntos (Fig. 1). Podemos observar que el nivel I obtiene menores puntuaciones que los niveles III y V. Se encontraron diferencias estadísticamente significativas entre el nivel I y ambos niveles superiores $(p<0,05)$. Podemos observar en la tabla II que la amplitud de las puntuaciones obtenidas fue mayor en el nivel I de ambas universidades.

$\mathrm{Al}$ analizar los datos en cada una de las universidades, observamos que el comportamiento de las puntuaciones en cada uno de los niveles es diferente. En la universidad 1 se observan puntuaciones más altas en relación con los años de carrera. Además, podemos observar en esta universidad que los datos tienden a una menor dispersión en los cursos mayores.

En la universidad 2 se observa que el nivel III es el de mayores puntuaciones y el nivel que obtiene menor dispersión de los datos (Tabla III y Fig. 2).

En relación con el género, se analizaron las puntuaciones en ambas universidades y también en cada una en forma independiente. En la figura 3 podemos observar que existe una distribución similar de las puntuaciones obtenidas entre hombres y mujeres. 


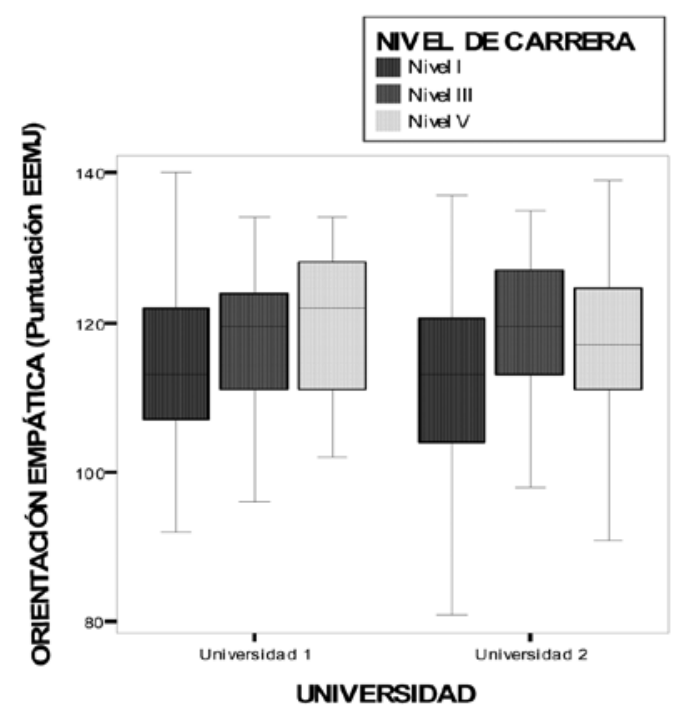

Figura 2. Puntuación obtenida respecto al nivel de carrera en cada una de las universidades.

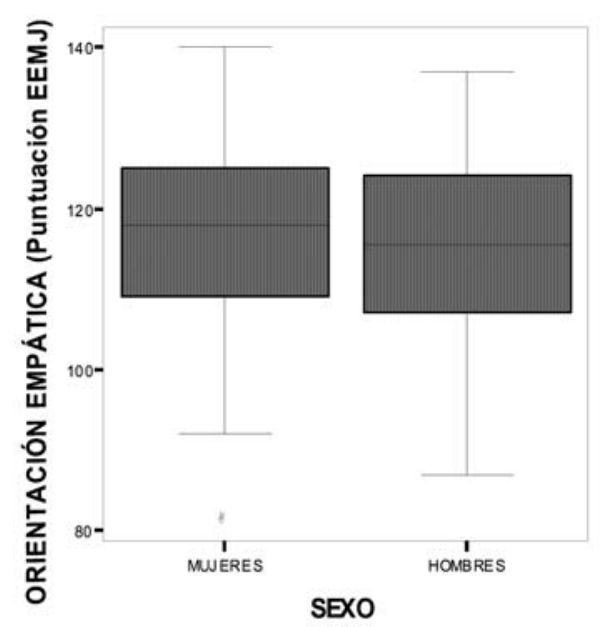

Figura 3. Puntuación obtenida en la escala de empatía médica de Jefferson respecto al sexo en ambas universidades.

Tabla III. Mediana y rango (mínimo y máximo) obtenidos por nivel en cada universidad.

\begin{tabular}{lcccc} 
& \multicolumn{2}{c}{ Universidad 1 } & \multicolumn{2}{c}{ Universidad 2 } \\
\hline Mediana & Rango & Mediana & Rango \\
\hline Nivel I & 113 & $92-140$ & 113 & $81-137$ \\
\hline Nivel III & 119,5 & $96-135$ & 119,5 & $98-135$ \\
\hline Nivel V & 121 & $102-134$ & 117 & $91-139$ \\
\hline
\end{tabular}

En cambio, al estudiar las puntuaciones en cada una de las universidades, vemos que en los hombres de la universidad 1 se observan diferencias entre los niveles que resultaron ser estadísticamente significativas entre ellas. Obtuvieron mayores puntuaciones los niveles más avanzados de formación, mientras que en la universidad 2 sólo se encontraron puntuaciones mayores con significación estadística en las mujeres del nivel III en relación con el nivel I $(p<0,05)$. Asimismo, observamos que el comportamiento de las puntuaciones obtenidas posee una distribución similar en ambas universidades (Fig. 4). Para el grupo de los hombres, se aprecian puntuaciones más altas en los cursos mayores de la carrera; en cambio, entre las mujeres, es el nivel III el que obtiene mayores puntuaciones.

\section{Discusión}

Los resultados obtenidos en este estudio nos permiten afirmar que las puntuaciones obtenidas en la EEMJ son mayores en los cursos más avanzados de la carrera. Esta diferencia es significativa 


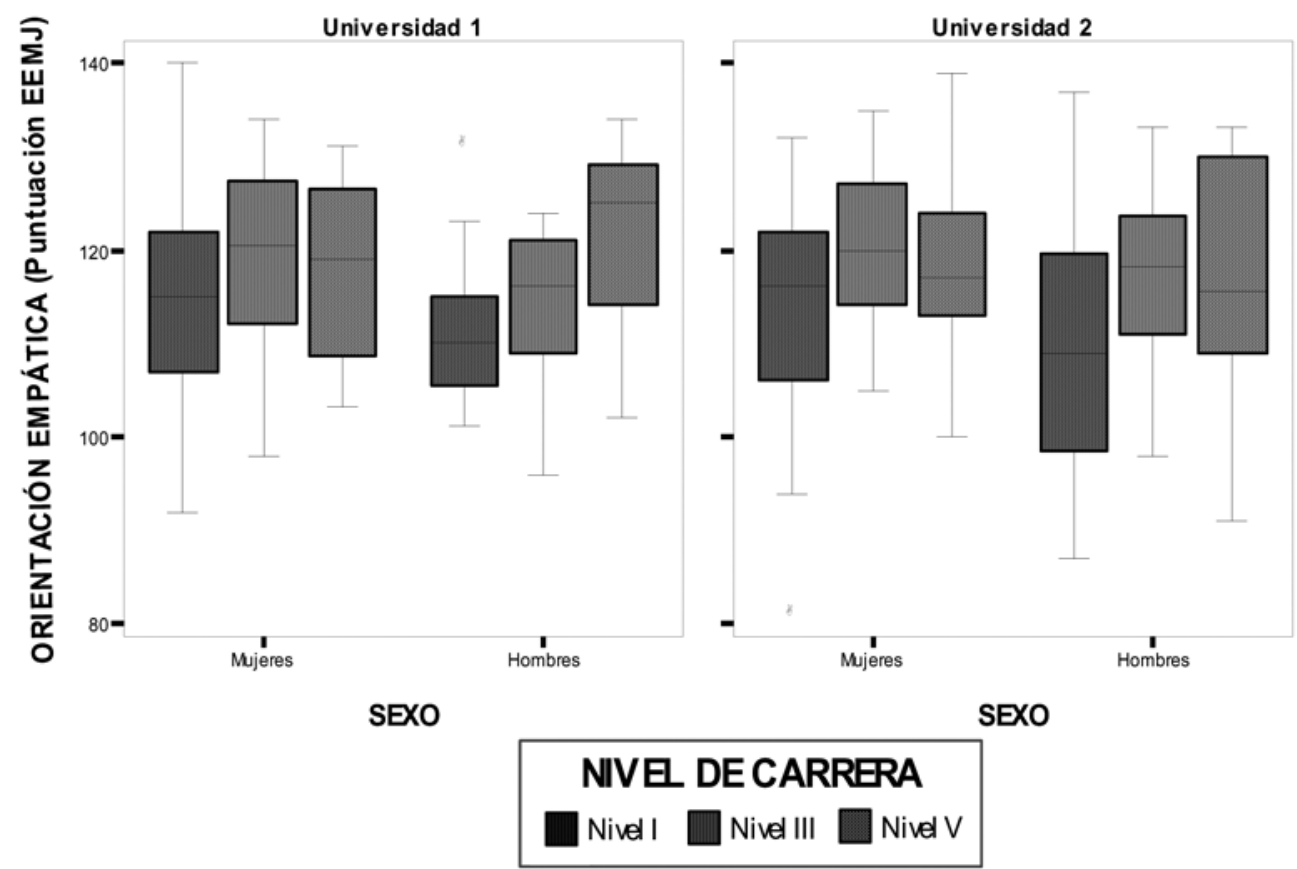

Figura 4. Puntuación obtenida en la escala de empatía médica de Jefferson respecto al sexo separado por nivel, en cada universidad.

en ambas universidades en conjunto, entre los niveles I-III y I-V.

Estos hallazgos no concuerdan con investigaciones similares que poseen un mismo modelo metodológico, en las cuales se plantea que la orientación empática va disminuyendo en los estudiantes de medicina a medida que avanzan en su carrera [4,11]. Otras investigaciones realizadas con estudiantes de odontología en los cuatro primeros años de formación muestran que el nivel I era el que tenía puntuaciones significativamente mayores de orientación empática con respecto a los demás niveles [8], lo que tampoco concuerda con las mayores puntuaciones observadas en esta investigación en los niveles más avanzados de carrera.

$\mathrm{Al}$ analizar las puntuaciones obtenidas en cada una de las universidades por separado, podemos observar que el rango entre las puntuaciones máximas y mínimas es mayor en el nivel I, mientras que en los niveles más elevados el rango es más pequeño. Pese a no ser objetivo principal de este estudio el análisis comparativo entre las dos escuelas participantes, es interesante observar que las puntuaciones tienden a tener una dispersión menor al avanzar en los niveles de carrera, aun- que esto se observa claramente sólo en una de las dos instituciones participantes; esto quiere decir que en el nivel I encontramos mayores diferencias en las puntuaciones obtenidas por los estudiantes para este mismo nivel, mientras que en el nivel V estas diferencias disminuyen.

Es interesante estudiar la hipótesis de que la tendencia a obtener mayores puntuaciones en los cursos superiores en ambas escuelas puede tener alguna relación con el tipo de formación que reciben los alumnos en carreras del área de la terapia física, donde el profesional debe pasar mucho tiempo con el paciente y donde el resultado de su terapia estará directamente vinculado con la relación que establezca con él. Además, estas carreras, en su mayoría, se enfrentan a la enfermedad desde un modelo biopsicosocial planteado por la nueva definición de la Organización Mundial de la Salud [15], lo cual permite que quienes tenemos la importante misión de enseñar en estas escuelas reconozcamos la importancia de los factores sociales y psicológicos en la rehabilitación de nuestros pacientes y le demos una importancia no menor a los temas de trato y comunicación tratante-paciente. 
Con respecto a las puntuaciones obtenidas en la EEMJ y el sexo en ambas universidades, las mujeres obtuvieron puntuaciones mayores que los hombres, sin tener esta diferencia significación estadística (Fig. 3). En el análisis de los resultados de ambas universidades por separado, se aprecia una evolución diferente de las puntuaciones obtenidas en la escala. Mientras que los hombres obtienen siempre puntuaciones mayores en los cursos superiores en ambas universidades, las mujeres tienden a mostrar puntuaciones menores en el último nivel de la carrera (Fig. 4).

Los resultados coinciden con varios trabajos en los cuales se ha aplicado el instrumento tanto en estudiantes como en residentes de medicina, $y$ en donde no hay diferencias significativas entre hombres y mujeres $[4,11,16]$. Otro estudio realizado a 704 médicos tampoco arrojó diferencias significativas en cuanto al sexo [3].

Una de las limitaciones de este trabajo, dada por su diseño metodológico, es que no permite observar cuál es la variación que experimenta la orientación empática a lo largo de los años de formación, ya que las mayores puntuaciones obtenidas en los niveles superiores podrían corresponder a una característica natural del grupo evaluado. Esto no permite concluir que la variable en estudio mejora o aumenta como resultado de la formación académica.

Por otro lado, el análisis por género en nuestro estudio está limitado, ya que la cantidad de hombres (el 32,12\% del total) es bastante menor que la de mujeres. Esto no nos permite inferir ninguna conclusión en relación con las diferencias de puntuaciones obtenidas por género, ya que se pueden deber simplemente a una coincidencia o a que la evolución de esta variable tiene un comportamiento diferente en ambos sexos.

Un factor que puede influir en las puntuaciones obtenidas y que es una variable desconcertante para los autores de este trabajo es el nivel de desarrollo personal de los estudiantes evaluados, ya que es posible encontrar en algunos cursos alumnos que ya son profesionales y que, además, han elegido estudiar kinesiología. Otra limitación de esta investigación es el estado de ánimo de los participantes en el momento de contestar la escala, lo que puede influir en los resultados obtenidos.

El hecho de que las universidades que formaron parte de este estudio sean diferentes, ya que la universidad 1 es pública y la universidad 2 es una entidad privada, podría significar algunas di- ferencias en el nivel socioeconómico, lo que podría influir en cierto modo el comportamiento de la variable estudiada; sin embargo, se observa un comportamiento ascendente relativamente similar en ambas escuelas.

Según los trabajos de Hojat et al $[4,5]$ y Sherman y Cramer [8], los alumnos experimentan una disminución en las puntuaciones obtenidas en la EEMJ al avanzar en la carrera, producto posiblemente de un estado de defensa que acompaña al miedo y la inseguridad que sienten los alumnos novatos cuando empiezan por primera vez a enfrentarse a pacientes $[8,11]$. En ambos trabajos, el enfrentamiento con responsabilidades directas con el paciente se produce en el segundo año de la carrera; en cambio, en nuestras escuelas los alumnos de primero a cuarto año ven pacientes de forma esporádica y sólo tienen responsabilidad sobre él en el quinto año de la carrera. Esto nos hace pensar que los alumnos de kinesiología en nuestro país se enfrentarían con mayor madurez y preparación a una situación clínica, lo que los haría menos susceptibles al temor y, por lo tanto, a enfrentar las situaciones de atención al paciente con mayor seguridad. Actualmente, la tendencia de los nuevos currículos de las carreras de la salud es incorporar las prácticas clínicas lo más temprano posible, idealmente desde los primeros años como un intento de mejorar las habilidades clínicas del estudiante. Sin embargo, parece ser absolutamente necesario preparar adecuadamente a los alumnos, tanto en el aspecto fisiopatológico y de las habilidades prácticas, como desde el punto de vista del enfrentamiento con el dolor y sufrimiento y el manejo de las relaciones interpersonales dadas en este contexto, para lograr de esta manera abordar la situación frente al paciente con un adecuado equilibrio emocional.

Creemos necesario investigar más aún sobre la orientación empática en el transcurso de la carrera, mediante la realización de estudios cualitativos que aborden a estudiantes y a pacientes en la situación de la práctica. Además, estudios longitudinales permitirían observar la evolución de la variable a lo largo del tiempo y relacionarla con estrategias formativas que permitan mejorar la orientación empática del alumnado.

Las relaciones interpersonales y las competencias clínicas comunicacionales son motivo de preocupación emergente dentro de las grandes asociaciones dedicadas a la formación de profesionales del área de la salud, y es urgente descu- 
brir los elementos que promueven el desarrollo de competencias comunicacionales y de estrategias de intervención que se pueden diseñar para la formación integral de profesionales de las distintas carreras implicadas en el cuidado de pacientes, más aún en aquellas relacionadas con la rehabilitación y el enfrentamiento con la discapacidad y las repercusiones que la misma genera en la vida de un ser humano.

\section{Bibliografía}

1. Fields SK, Hojat M, Gonnella JS, Mangione S, Kane G. Comparison of nurse and physicians on an operational measure of empathy. Eval Health Prof 2004; 27: 80-94.

2. Hojat M, Mangione S, Nasca TJ, Cohen MJM, Gonnella JS, Erdmann JB, et al. The Jefferson Scale of Physician Empathy: development and preliminary psychometric data. Educ Psychol Meas 2001; 61: 349-65.

3. Hojat M, Gonnella JS, Nasca TJ, Mangione S, Vergare M, Magee M. Physician empathy: definition, components, measurement, and relationship to gender and specialty. Am J Psychiatry 2002; 159: 1563-9.

4. Hojat M, Mangione S, Nasca TJ, Rattner S, Erdmann JB, Gonnella JS, et al. An empirical study of decline in empathy in medical school. Med Educ 2004; 38: 934-41.

5. Hojat M, Mangione S, Nasca TJ, Gonnella JS, Magee M. Empathy scores in medical school and ratings of empathic behavior in residency training 3 years later. J Soc Psychol 2005; 145: 663-72.

6. Alcorta-Garza A, González JF, Tavitas S, Rodríguez FJ, Hojat M. Validación de la escala de empatía médica de
Jefferson en estudiantes de medicina mexicanos. Salud Mental 2005; 28: 57-63.

7. Jackson PL, Meltzoff AN, Decety J. How do we perceive the pain of others? A window into the neural processes involved in empathy. Neuroimage 2005; 24: 771-9.

8. Sherman JJ, Cramer A. Measurements of changes in empathy during dental school. J Dent Educ 2005; 69: 338-45.

9. Boada M, Méndez A. Caracterización de los reclamos del sector salud recibidos en el Servicio Nacional del Consumidor. Chile, enero 2004-marzo 2005 [tesis de licenciatura]. Santiago: Escuela de Kinesiología, Universidad de Chile; 2005.

10. Maheux B, Belant F. Students' perception of values emphasized in three medical schools. Acad Med 1986; 61: 308-16.

11. Mangione S, Kane GC, Caruso JW, Gonnella JS, Nasca TJ, Hojat M. Assessment of empathy in different years of internal medicine training. Med Teach 2002; 24: 371-4.

12. Hojat M, Gonnella JS, Mangione S, Nasca TJ, Veloski JJ, Erdmann JB, et al. Empathy in medical students as related to academic performance, clinical competence, and gender. Med Educ 2002; 36: 1-6.

13. Hojat M, Gonnella J, Nasca TJ, Mangione S, Veloski JJ, Magee M. The Jefferson Scale of Physician Empathy: further psychometric data and differences by gender and specialty at item level. Acad Med 2002; 77: S58-60.

14. Hojat M, Fields SK, Gonnella JS. Empathy: an NP/MD comparison. Nurse Pract 2003; 28: 45-7.

15. Egea C, Sarabia A. Clasificaciones de la OMS sobre discapacidad. Boletín del Real Patronato sobre Discapacidad 2001; 50: 15-30.

16. Carvajal A, Miranda CI, Martinac T, García C, Cumsille F. Análisis del nivel de empatía en un curso de quinto año de medicina, a través de una escala validada para este efecto. Revista del Hospital Clínico de la Universidad de Chile 2004; 10: 302-6. 